\title{
A Method to Calibrate the Electromagnetic Tracking Instrument When Measuring Branches of Fruit Trees
}

\author{
Ding-Feng Wu, Jian Wang, Guo-Min Zhou, and Li-Bo Liu \\ Agricultural information institute of CAAS, Beijing 100081, China \\ wonderful_webstudio@hotmail.com
}

\begin{abstract}
To reduce the effect from instrument error when getting characteristic parameters of branches of fruit trees by the electromagnetic tracking instrument, a calibration method was sounded based on a discussion of the instrument error of electromagnetic tracking instrument. Finally, the method was tested in an experiment. By comparing the data of the experiment and the standard data which was got by slide caliper, we proved that the method is effective in increasing the accuracy of measurement.
\end{abstract}

Keywords: Fastrak; Instrument error; Calibration.

\section{Introduction}

China is the biggest producer of fruits in the world. In many parts of this country, the fruit industry has become the pillar industry. based on the measurement of fruit tree structure, the research of the connection between the structure and the output, the utility rate of luminous energy and the anti-disease ability of a fruit tree is an important impetus of developing of punning skill and breeding technique of fruit trees [4].

Electromagnetic tracking instrument is a kind of digital measuring tools based on electromagnetism [3]. It is an effective tool of getting structure data of fruit trees because it is not only an easy-to-use, extremely accurate and broad action sphere device but also a powerful survey tool which can track the space track and calculate the inclination angle of stylus [2].

The electromagnetic tracking instrument is vulnerable to external magnetic effects. It will fall in complicated electromagnetic environment [1]. Besides, after a long time working, the status of equipment will be different from the initial status and the accuracy of the device will reduce. When measuring branches of fruit trees, a high degree of accuracy is required, so the electromagnetic tracking instrument must be calibrated before working [4]. In this paper, a calibration method is put forward.

\section{Materials and Methods}

\subsection{Device}

Fastrak is an advanced electromagnetic tracking instrument [1]. It was used as the measuring device in the experiment. 
Assume the error of the measuring instrument to be calibrated is $\mu$ and the error of the standard measuring device is $\mu$ '. Then in the course of the instrument calibration, we must ensure that $\mu$ ' is at a lower order of magnitude than $\mu$, otherwise the calibration may increase the error because the error of standard device affects the result. As we known, the Fastrak electromagnetic tracking device can working with accuracy of $0.8 \mathrm{~mm}$ [1], which means the normal rulers can not provide a standard Reference Data, so we use a slide caliper with the precision of $0.05 \mathrm{~mm}$ as the standard measuring device in the experiment.

\subsection{Analysis of Error}

Because of the effects of devices and experiment environment, the measurement result of physical amount is definitely different with the real value, the difference is called measurement error, the part which caused by the imperfect instrument structure and the external environment is named instrument error.

When Fastrak is working, following causes may bring instrument error:

1. External magnetic effects

2. Deviation of origin of coordinate

3. Instrument mechanical wear and decline of circuit state

\subsection{Error under the Magnetic Effects}

In the electromagnetic environment, eight space points were measured by Fastrak electromagnetic tracking instrument, every point was measured ten times. The result was compared with the standard data got by slide caliper. Error of one point's ten times measurement is shown in Figure 1.

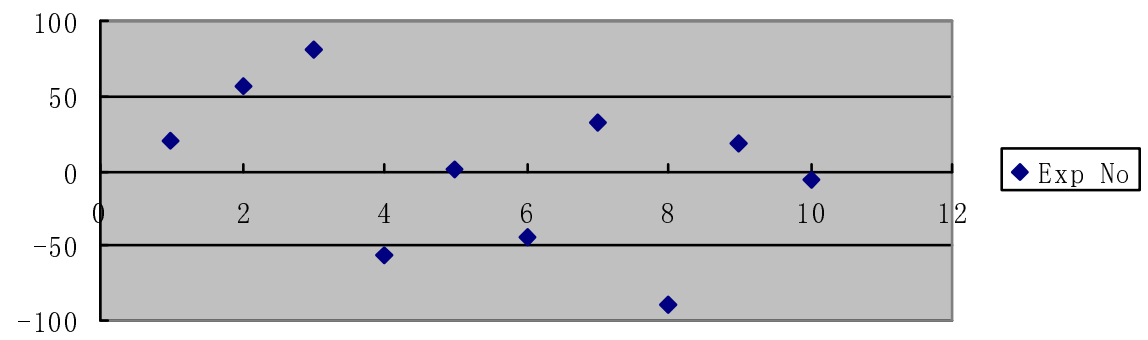

Fig. 1. Randomness of electromagnetic tracking instrument's error in electromagnetic environment

As shown in Figure 1, in electromagnetic environment, Fastrak's error is random and irregular. So the error brought by external magnetic effects is hard to reduce by calibration, the calibration should be conducted without the effect of external magnetic. 


\subsection{Calibration Method}

Assume the initial space coordinates of origin are $(\mathrm{x}, \mathrm{y}, \mathrm{z})$, after the deviation, the coordinates are changed to ( $\left.x^{\prime}, y^{\prime}, z^{\prime}\right)$, the amounts of deviation are $\Delta x, \Delta y$ and $\Delta z$, so $\Delta \mathrm{x}=\mathrm{x}^{\prime}-\mathrm{x}, \Delta \mathrm{y}=\mathrm{y}^{\prime}-\mathrm{y}, \Delta \mathrm{z}=\mathrm{z}^{\prime}-\mathrm{z}$. When a space point is measured by the measuring device, assume the coordinates of the point got by measuring device are $(\mathrm{X}, \mathrm{Y}, \mathrm{Z})$, then the real coordinates are $\left(\mathrm{X}^{\prime}-\Delta \mathrm{x}, \mathrm{Y}^{\prime}-\Delta \mathrm{y}, \mathrm{Z}^{\prime}-\Delta \mathrm{z}\right)$. As we known, $\Delta \mathrm{x}, \Delta \mathrm{y}$ and $\Delta \mathrm{z}$ are constants, so we just need to use the above method to $\mathrm{n}$ space points to get their $\Delta \mathrm{x}_{\mathrm{i}}$, $\Delta \mathrm{y}_{\mathrm{i}}, \Delta \mathrm{z}_{\mathrm{i}}(\mathrm{i} \in[1, \mathrm{n}]), \Delta \mathrm{x}, \Delta \mathrm{y}$ and $\Delta \mathrm{z}$ can be calculated by following formulas:

$$
\begin{aligned}
& \Delta \mathrm{x}= \frac{\sum_{i=1}^{n} \Delta \mathrm{x}_{\mathrm{i}}}{n} \\
& \Delta \mathrm{y}=\frac{\sum_{i=1}^{n} \Delta \mathrm{y}_{\mathrm{i}}}{n} \\
& \Delta \mathrm{z}=\frac{\sum_{i=1}^{n} \Delta z_{i}}{n}
\end{aligned}
$$

The other part of instrument error can be parted into two parts: one has the relationship with the distance between the space point and the transmitter of Fastrak, the other one is a constant. Assume they are $\mu 1$ and $\mu 2 . \mu 2$ is a constant, so it can be reduced by the same method as the method reducing the error brought by origin deviation. Assume the distance between the space point and the transmitter of Fastrak is $L$, then $\mu 1=f(L)$. In theory, when $\mathrm{L}$ is $0, \mathrm{f}(\mathrm{L})$ is going to be 0 , but in actual use of Fastrak, as shown in

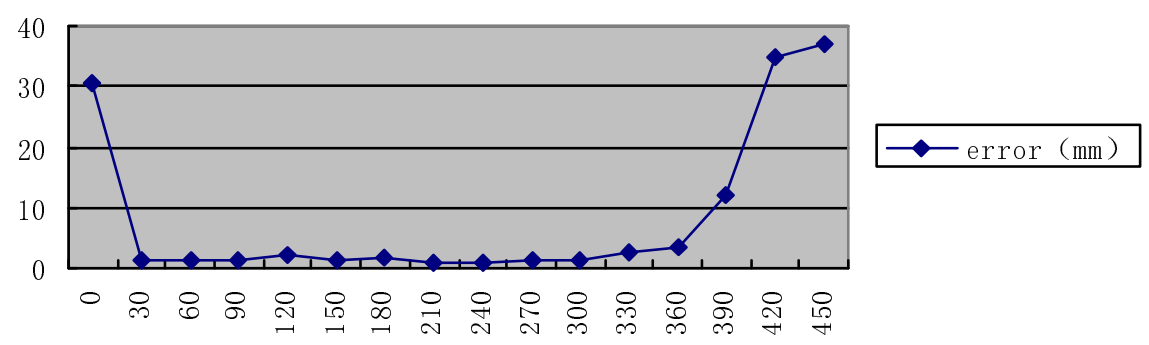

Fig. 2. Relationship between error and distance between space point and transmitter 
Figure 2, when L was enough small, the time of signal transmitting was too short to be accurately measured by the device, as a result, it brought in an un-negligible error, so the calibration can only reduce the constant error.

The calibration method is shown in Figure 3.

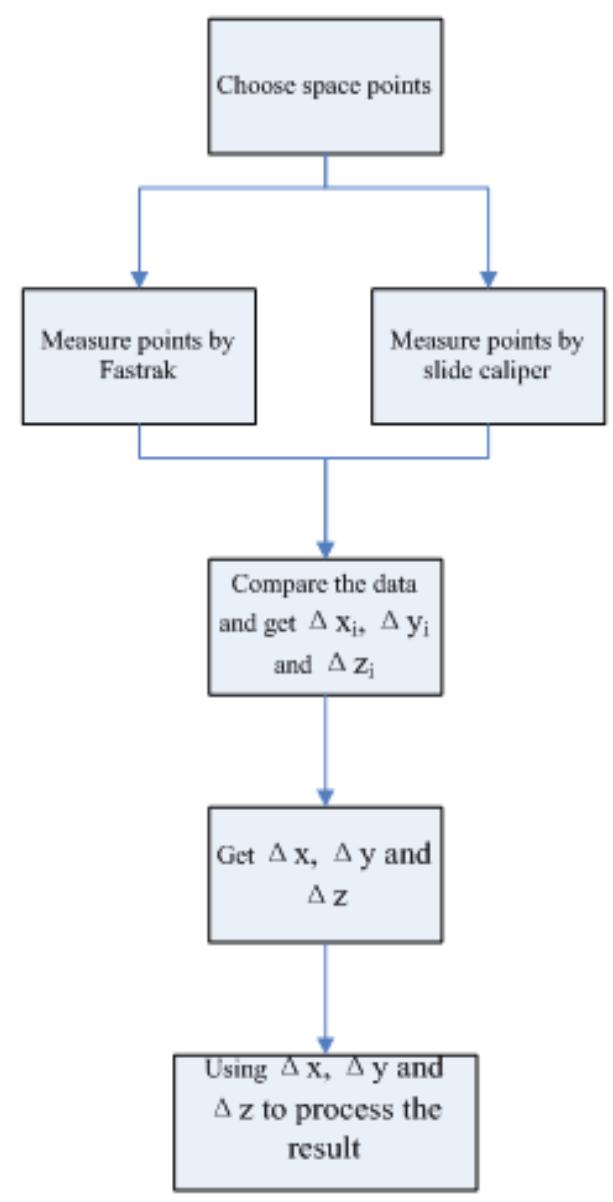

Fig. 3. Flow chart of Calibration method

\subsection{Experiment}

We got one space point in each quadrant of the eight quadrants conformed by the Spatial three dimensional coordinate axis and one space point on each axis, so we had eleven points which were measured in the experiment. Those points were measured by Fastrak electromagnetic tracking instrument. The result of the measurement was processed by the above calibration method. At the end of the experiment, we compared the result with the standard data got by slide caliper. 


\section{Result and Analysis}

The experiment result is shown in Figure 4, Figure 5 and Figure 6.
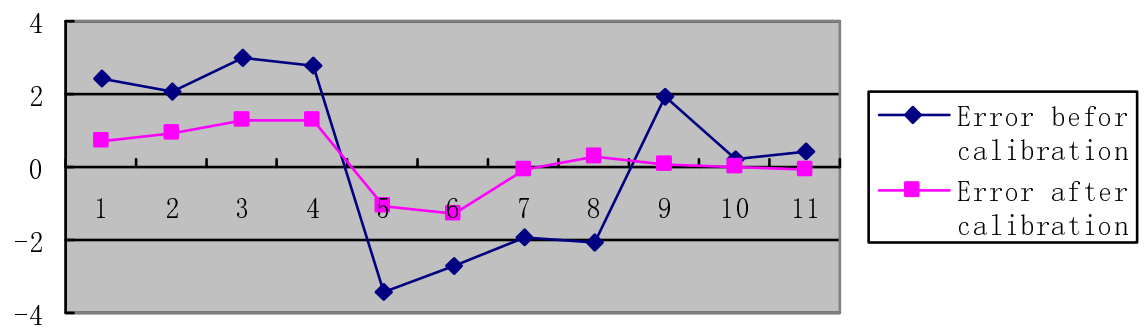

Fig. 4. The compare of the error before and after the calibration (the $\mathrm{X}$ axis)
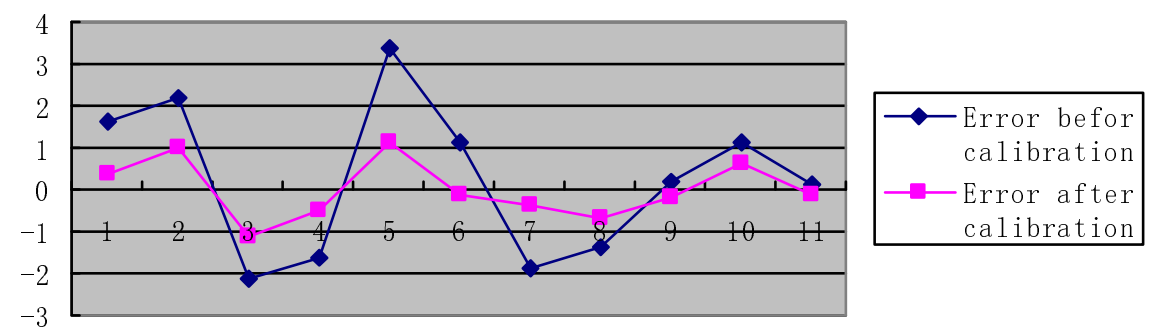

Fig. 5. The compare of the error before and after the calibration (the $\mathrm{Y}$ axis)
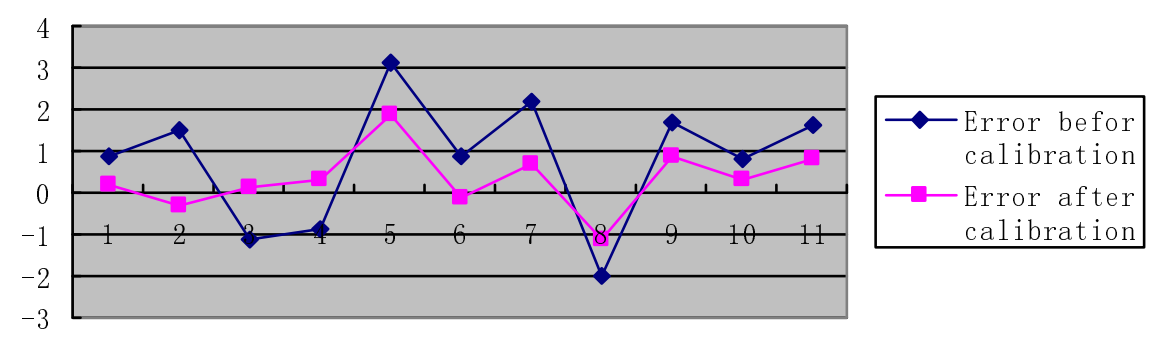

Fig. 6. The compare of the error before and after the calibration (the $\mathrm{Z}$ axis)

It is clear that the errors after the calibration were less than the errors before the calibration. It proved that the method does work.

\section{Conclusion and Discussion}

Based on the analysis of causes and characters of the error of the electromagnetic tracking instrument, a calibration method was given, and then an experiment proved the availability of the method. The method can improve the accuracy when measuring branches of fruit trees by the electromagnetic tracking instrument. 


\section{References}

1. Polhemus: 3Space Fastrak Users Manual (2000)

2. Ivanov, N., Boissard, P., Chapron, M., Valery, P.: Estimation of the Height and Angles of Orientation of the Upper Leaves in the Maize Canopy Using Sterovision. Agrononie (1994)

3. Danjon, F., Sinoquet, H., Godin, C., et al.: Characterisation of structural tree root architecture using 3D digitising and AMAP mod software. Plant and soil (1999)

4. Thanisawanyangkura, S., Sinoquet, H., River, P., et al.: Leaf orientation and sunlit leaf area distribution in cotton. Agricultural and Forest Meteorology (1997) 